\title{
Obtención de un aglomerado para material de construcción a partir de un adhesivo obtenido de viruta de wet blue del proceso del curtido de pieles.
}

Obtaining an agglomerate for construction material from an adhesive obtained from wet blue chip from the leather tanning process.

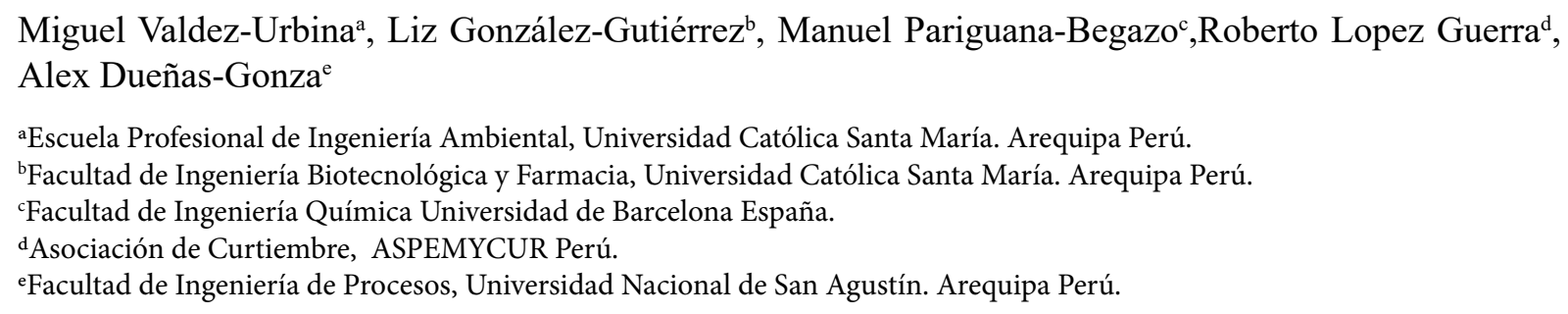

\section{INFORMACIÓN}

\section{Historia del Artículo}

Recepción: 10/01/2019

Revisión: 29/04/2019

Aceptación: 02/05/2019

\section{Palabras Clave}

Aglomerado, adhesivo, viruta, densidad, conductividad, densidad, construcción.

\section{Key Words}

Agglomerate, adhesive, chip, density, conductivity, density, construction.

\section{DOI}

https://doi.org/10.35286/veritas. v20i1.233

\begin{abstract}
RESUMEN
La industria del curtido de pieles produce una cantidad grande de aguas residuales y desperdicios sólidos que contienen una cantidad significativa de cromo, la viruta de wet blue es un material particulado sólido microcolagenosas impregnado con grasa y aceite sintético de cromo y agentes curtientes. El objetivo del estudio fue investigar la producción de un aglomerado a partir de un adhesivo obtenido de la viruta de wet blue producida mediante hidrólisis alcalina a $60^{\circ} \mathrm{C}$ de temperatura, $0.4 \mathrm{~N}$ de $\mathrm{NaOH}$ y 4 hrs. para la neutralización. Para la obtención del aglomerado se utilizó $2 \mathrm{Kg}$ de aserrín de $1 \mathrm{~mm}$ de madera de ébano el que secó a $105^{\circ} \mathrm{C}$ por 4 horas, posteriormente se realizó un mezclado manual en dos proporciones; $125 \mathrm{gr}$ de adhesivo y 250 gr de aserrín (0.5/1), y una segunda proporción de $250 \mathrm{gr}$ de adhesivo y $250 \mathrm{gr}$ de aserrín $(1 / 1)$, las mezclas se realizaron por 20 minutos a temperatura ambiente, el prensado fue al frio a $100 \mathrm{~kg} / \mathrm{cm} 2$, en moldes de acero inoxidable para evitar que se pegue el aglomerado, las muestras permanecieron en la prensa por 2 hrs., terminado el prensado las muestras fueron expuestas al medio ambiente en un lugar con incidencia solar, donde permanecieron en el molde por las siguientes $48 \mathrm{hrs}$. Al culminar este tiempo las muestras se retiraron del molde para ser expuestas al sol por 5 días, el aglomerado obtenido fue sometido a pruebas mecánicas tales como la resistencia a la compresión en una máquina de ruptura universal de acuerdo a la norma DIN 68763 para "tableros planos de partículas para uso en conceptos de construcción de edificios, requisitos, pruebas e inspección", también fueron sometidos a la prueba de conductividad térmica de acuerdo a la norma ASTM C-177 en un gradiente térmico emitido por una plancha de 1200 watts, midiendo la temperatura pasante cada 5 minutos, y la Prueba de densidad aparente de acuerdo a la norma DIN 68763. Los resultados mostraron en las pruebas de conductividad térmica que la mezcla $1 / 1$ (250gr de adhesivo y 250 gr de aserrín),presentó menor promedio que la norma ASTM C-177 $(0.28 \mathrm{~W} / \mathrm{m} . \mathrm{K})$ con un promedio de $0.22 \mathrm{~W} / \mathrm{m} . \mathrm{K}$, además esta mezcla presentó mayor resistencia a la compresión que la norma DIN $68763(175 \mathrm{Kgf} / \mathrm{m} 2)$ con un promedio de $291.57 \mathrm{Kgf} / \mathrm{m} 2$, demostrando ser un aglomerado muy resistente para construcción y representando una solución ecoamigable para la disposición final de la viruta wet blue, del proceso del curtido de pieles.
\end{abstract}

\begin{abstract}
The leather tanning industry produces a large amount of wastewater and solid waste containing a significant amount of chromium, the wet blue chip is a solid micro-cocoa particulate material impregnated with chromium synthetic oil and grease and tanning agents. The objective of the study was to investigate the production of an agglomerate from an adhesive obtained from the wet blue chip produced by alkaline hydrolysis at $60^{\circ} \mathrm{C}$ temperature, $0.4 \mathrm{~N} \mathrm{NaOH}$ and 4 hrs. for neutralization. To obtain the agglomerate, $2 \mathrm{~kg}$ of sawdust of $1 \mathrm{~mm}$ of ebony wood was used, which was dried at $105^{\circ} \mathrm{C}$ for 4 hours, then manual mixing was carried out in two proportions; $125 \mathrm{~g}$ of adhesive and $250 \mathrm{~g}$ of sawdust $(0.5 / 1)$, and a second proportion of 250 $\mathrm{g}$ of adhesive and $250 \mathrm{~g}$ of sawdust (1/1), the mixtures were carried out for 20 minutes at room temperature, the pressing was cold at $100 \mathrm{~kg} / \mathrm{cm} 2$, in stainless steel molds to avoid sticking the agglomerate, the samples remained in the press for $2 \mathrm{hrs}$., after the pressing the samples were exposed to the environment in a place with solar incidence, where they remained in the mold for the next $48 \mathrm{hrs}$. At the end of this time the samples were removed from the mold to be exposed to the sun for 5 days, the agglomerate obtained was subjected to mechanical tests such as the compressive strength in a universal breaking machine according to DIN 68763 for "boards particle plans for use in concepts of building construction, requirements, testing and inspection
\end{abstract}




\begin{abstract}
", were also subjected to the thermal conductivity test according to ASTM C-177 in a thermal gradient emitted by a 1200 -watt plate, measuring the temperature passed every 5 minutes, and the apparent density test according to DIN 68763. The results showed in the thermal conductivity tests that the mixture $1 / 1$ (250gr of adhesive and $250 \mathrm{~g}$ of sawdust), presented lower average than the ASTM C-177 standard $(0.28 \mathrm{~W} / \mathrm{mK})$ with an average of $0.22 \mathrm{~W} / \mathrm{mK}$, in addition this mixture presented higher compressive strength than DIN $68763(175 \mathrm{Kgf} / \mathrm{m} 2)$ with a an average of $291.57 \mathrm{Kgf} / \mathrm{m} 2$, proving to be a very resistant agglomerate for construction and representing an eco-friendly solution for the final disposal of the wet blue chip, from the leather tanning process.
\end{abstract}

\section{INTRODUCCIÓN}

La industria del curtido de pieles produce gran cantidad de desechos sólidos y en especial las "Virutas de Wet Blue" que constituyen el $25 \%$ de los sólidos eliminados, compuestas por el complejo colágeno-Cr (Alfredo Schneider et al, 2012). Por ello, se disponen en reservorios especiales, con costo adicional.

La industria de la curtiembre es considerada de alto impacto, ya sea en lo social, por los puestos de trabajo que genera, en lo económico, por la cantidad de divisas que moviliza, y sobre todo en lo ambiental, por los desechos que produce (Hoinacki et al., 1994). Sus constituyentes, colágeno (proteína) y $\mathrm{Cr}$, sugieren el desarrollo de un material aislante aprovechando la baja conductividad térmica del cuero, y el poder adhesivo de las "colas" producto de la hidrólisis del colágeno; incluyendo muchas veces como carga las virutas y como adhesivo los productos de la reacción de las virutas del wet blue, previa separación por extracción de pequeñas cantidades de péptidos (Schneider et al., 2009). Para caracterizar estos materiales aglomerantes, se necesita conocer sus propiedades y la forma en que influyen las diferentes variables de operación sobre las mismas. Esta investigación incluirá la elaboración de un material aglomerante a base de un adhesivo de viruta de wet blue y aserrín de madera de tornillo, para evaluar su resistencia a la compresión, conductividad térmica y densidad aparente.

\section{METODOLOGÍA}

\section{Obtención de las muestras}

La muestra correspondiente a residuos del proceso de raspado del cuero viruta de Wet Blue se obtuvo de una empresa curtidora de pieles, ubicada dentro del Parque industrial de Rio Seco del distrito de Cerro Colorado, y las muestras de aserrín de madera tornillo y caoba de una carpintería de la zona de Zamácola de la ciudad de ArequipaPerú.

\section{Obtención del aglomerado}

Para la obtención del aglomerado Para la caracterización se seleccionó una muestra inicial de $200 \mathrm{~kg}$ de viruta de Wet blue hidrolizada a $4 \mathrm{~N}$ de $\mathrm{NAOH}, 60^{\circ} \mathrm{C}$ y $4 \mathrm{Hrs}$, mediante el método del cuarteo referente a residuos sólidos descrito en la guía metodológica para el desarrollo del estudio de caracterización de residuos sólidos municipales (ECRSM) hasta la obtención de una muestra final de 100 grs., para analizar el porcentaje de proteínas, concentración de cromo y contenido de humedad. La fabricación del material aglomerado se produce mezclando en distintas proporciones el aglomerante (producto de la hidrólisis) y la carga (virutas).
El aglomerado obtenido se seca, y luego se ensaya a fines de evaluar su aptitud para el fin propuesto (Landrock,1985).

\section{Adecuación del aserrín}

La muestra de $1 \mathrm{Kg}$. de aserrín fue extraída por el método del cuarteo, para su molienda en cuchillas y posterior tamizaje con malla 100 hasta obtener un tamaño uniforme de $1 \mathrm{~mm}$.

\section{Reducción de la humedad}

Se pesó 500 gr de aserrín, los que se secaron a $105^{\circ} \mathrm{C}$ por 4 horas en una estufa $\mathrm{Al}$ culminar la reducción de humedad se procederá muy rápido al mezclado ya que el aserrín puede volver a absorber la humedad del medio ambiente.

\section{Mezclado}

Para esta fase se utilizó un mezclado manual que consta en dos grupos; el primer grupo se colocó en un recipiente 250 gr de aserrín y 125 gr de adhesivo, y el segundo grupo se colocó 250 gr de aserrín y 250 gr de adhesivo. Estos dos grupos fueron mezclados por 20 minutos con una paleta de palo a temperatura ambiente.

\section{Prensado}

Para el prensado se utilizó la técnica de prensado en frio, la prensa usada para este procedimiento tiene una fuerza de presión de hasta $100 \mathrm{~kg} / \mathrm{cm} 2$ y es manual. Los moldes empleados son de acero inoxidable para evitar que se pegue el aglomerado. La masa obtenida se vacía hasta la señalización del molde, posteriormente se empieza aplicar la presión hasta que no se pueda ejercer más fuerza. Las muestras permanecerán en la prensa por dos horas posteriormente serán retiradas.

\section{Secado}

Al culminar la etapa de prensado las muestras fueron expuestas al medio ambiente en un lugar donde se encuentre gran incidencia solar, permanecerán en el molde por las siguientes 48 horas. Al culminar este tiempo las muestras se retiran del molde para ser expuestas al sol por los próximos 5 días terminando así la realización del aglomerado sin acabado.

\section{Pruebas mecánicas y físicas al aglomerado}

Al obtener un aglomerado este necesita pasar por distintas pruebas mecánicas las cuales demostraran su posible utilidad, en este estudio se eligieron tres pruebas básicas desarrolladas a continuación. Las pruebas se le realizaron a los dos tipos de aglomerado obtenido. 


\section{Prueba de resistencia a la compresión}

La resistencia a la compresión del aglomerado, es uno de los parámetros más importantes para evaluar sus características como elementos de construcción. Los resultados de este ensayo se compararon con la norma DIN 68763 "tableros planos de partículas para uso en conceptos de construcción de edificios, requisitos, pruebas e inspección".

Para este ensayo se utilizó una máquina de ruptura universal, primero se tomaron las medidas exactas de las probetas al saber esto se coloca estas probetas de forma paralela a su fibra, posteriormente se empieza el ensayo con el aumento de carga hasta llegar a observar la falla donde se detiene el estado de carga y se hace su lectura.

\section{Prueba de conductividad térmica}

La prueba de conductividad térmica demostrara si es un material aislante y su resistencia a la combustión, esta se realiza según la norma ASTM C-177 Standard Method for Steady State Thermal Propeties by means of the Guarded Hot Plate, a su vez los resultados serán comparados con Norma Chilena Oficial NCh. 853.93 “ conductividad termica de materiales " El ensayo consistió en someter las probetas de dimensiones conocidas (área y espesor) a un gradiente térmico emitido por una plancha de 1200 watts, midiendo la temperatura pasante cada 5 minutos. Teniendo estos datos se ingresaran a la fórmula de Fourier para obtener la conductividad térmica.

$$
K=\frac{Q * L}{A * \Delta T}
$$

\section{Prueba de densidad aparente}

Para la evaluación de los resultados se comparará con la norma DIN 68763 establecida para "tableros planos de partículas para uso en conceptos de construcción de edificios, requisitos, pruebas e inspección”.

\section{RESULTADOS Y DISCUSIÓN}

Determinación de la resistencia a la compresión del aglomerado

Los resultados de la resistencia a la compresión del aglomerado mostraron diferencias altamente significativas $(\mathrm{p}<0.01)$, para ambas mezclas, siendo la mezcla con relación 1 parte adhesivo de viruta de wet blue +1 aserrín de madera de tornillo con a que se obtuvo mayor resistencia a la compresión con un promedio de $291.57 \mathrm{Kgf} / \mathrm{cm} 2$ (tabla 1), además los valores promedio de las pruebas de compresión comparadas con el valor mínimo establecido por la norma DIN 68763 (valores recomendados para aglomerados como material de construcción) $175 \mathrm{Kfg} / \mathrm{m} 2$ resultaron ser mayores.
Tabla 1: Comparación de la resistencia a la compresión según mezcla del aglomerado.

\begin{tabular}{|c|c|c|c|c|}
\hline \multirow{3}{*}{ VARIABLE } & \multicolumn{2}{|c|}{$\begin{array}{l}\text { MEZCLA DEL } \\
\text { AGLOMERADO }\end{array}$} & \multirow{3}{*}{$\mathrm{t}$} & \multirow{3}{*}{$\mathrm{P}$} \\
\hline & $\begin{array}{c}\text { (0.5 adhesivo }+ \\
1 \text { aserrín) }\end{array}$ & $\begin{array}{c}(1 \text { adhesivo }+1 \\
\text { aserrín) }\end{array}$ & & \\
\hline & $\bar{x} \pm S$ & $\bar{x} \pm s$ & & \\
\hline Espesor & $1.04 \pm 0.02$ & $1.06 \pm 0.02$ & 1.4 & $0.234(\mathrm{p}>0.05)$ \\
\hline Ancho & $7.55 \pm 0.01$ & $7.50 \pm 0.02$ & 5.3 & $0.006(\mathrm{p}<0.01)$ \\
\hline Peso inicial & $46.57 \pm 0.01$ & $49.21 \pm 0.04$ & 108.79 & $\begin{array}{l}0.000 \ldots \\
(\mathrm{p}<0.01)\end{array}$ \\
\hline Peso seco & $40.36 \pm 0.03$ & $43.75 \pm 0.08$ & 68.97 & $\begin{array}{l}0.000 \ldots \\
(\mathrm{p}<0.01)\end{array}$ \\
\hline $\begin{array}{l}\text { Contenido } \\
\text { humedad }\end{array}$ & $13.32 \pm 0.09$ & $11.09 \pm 0.16$ & 21.81 & $\begin{array}{l}0.000 \ldots \\
(\mathrm{p}<0.01)\end{array}$ \\
\hline $\begin{array}{c}\text { Resistencia a } \\
\text { la compresión } \\
(\mathrm{Kgf} / \mathrm{cm} 2)\end{array}$ & $200.13 \pm 4.08$ & $291.57 \pm 2.81$ & 31.98 & $\begin{array}{c}0.000 \ldots \\
(p<0.01)\end{array}$ \\
\hline
\end{tabular}

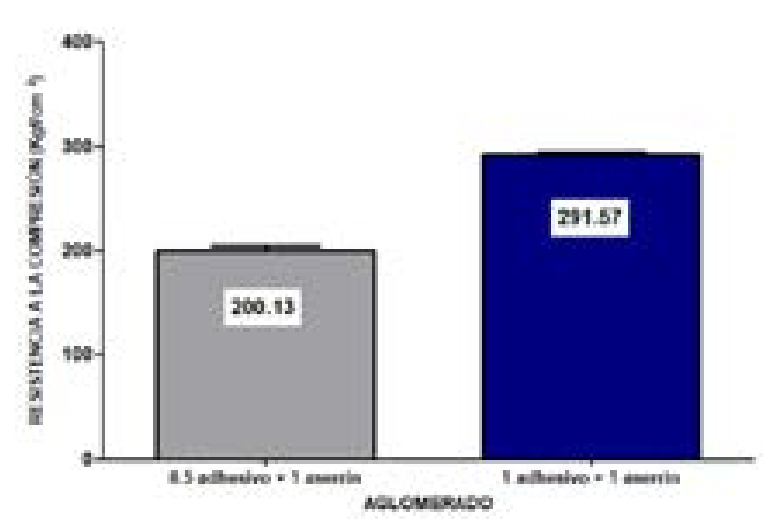

Fig. 1: Resistencia a la compresión según mezcla del aglomerado. Se muestra que la resistencia a la compresión del aglomerado del adhesivo para la mezcla 0.5 de adhesivo de viruta de wet blue más una parte de aserrín de tornillo presentó $200.13 \mathrm{Kgf} / \mathrm{cm} 2$, y la mezcla 1 parte del adhesivo de viruta de wet blue más una parte de aserrín de y la mezcla 1 parte del adhesivo
tornillo presentó $291.57 \mathrm{Kgf} / \mathrm{cm} 2$

Determinación de la conductividad térmica del aglomerado

La conductividad térmica presentó diferencias altamente significativas $(\mathrm{p}<0.01)$, para las mezclas del aglomerado, siendo los valores promedios de la conductividad térmica de las mezclas probadas mayores al valor límite de conductividad térmica $0.28 \mathrm{~W} / \mathrm{m} . \mathrm{K}$ dispuesto por la norma Nch 853.9, este resultado se puede atribuir en parte al contenido de cromo que contiene (tabla 2). 
Tabla 2: Comparación de la conductividad térmica según mezcla del aglomerado.

\begin{tabular}{|c|c|c|c|c|}
\hline \multirow{3}{*}{ VARIABLE } & \multicolumn{2}{|c|}{$\begin{array}{l}\text { MEZCLA DEL } \\
\text { AGLOMERADO }\end{array}$} & \multirow{3}{*}{$\mathrm{t}$} & \multirow{3}{*}{$\mathrm{P}$} \\
\hline & $\begin{array}{c}\text { (0.5 adhesivo }+ \\
1 \text { aserrín })\end{array}$ & $\begin{array}{l}\text { (1 adhesivo }+1 \\
\text { aserrín) }\end{array}$ & & \\
\hline & $\bar{x} \pm s$ & $\bar{x} \pm s$ & & \\
\hline Q & $98.00 \pm 0,00$ & $98.00 \pm 0,00$ & - & - \\
\hline Espesor & $0.01 \pm 0,00$ & $0.01 \pm 0,00$ & - & - \\
\hline $\begin{array}{l}\text { Área de } \\
\text { contacto }\end{array}$ & $0.04 \pm 0,00$ & $0.04 \pm 0,00$ & - & - \\
\hline $\begin{array}{l}\text { Temperatura } \\
\text { irradiada }\end{array}$ & $145.00 \pm 2.00$ & $146.33 \pm 2.08$ & 0.80 & $0.469(\mathrm{p}>0.05)$ \\
\hline $\begin{array}{l}\text { Temperatura } \\
\text { pasante }\end{array}$ & $49.67 \pm 3.06$ & $39.00 \pm 3.61$ & 3.91 & $0.017(\mathrm{p}<0.05)$ \\
\hline $\begin{array}{l}\text { Conductividad } \\
\text { térmica } \\
(\mathrm{W} / \mathrm{m} . \mathrm{k})\end{array}$ & $0.25 \pm 0.01$ & $0.22 \pm 0.01$ & 4.78 & $0.009(\mathrm{p}<0.01)$ \\
\hline
\end{tabular}

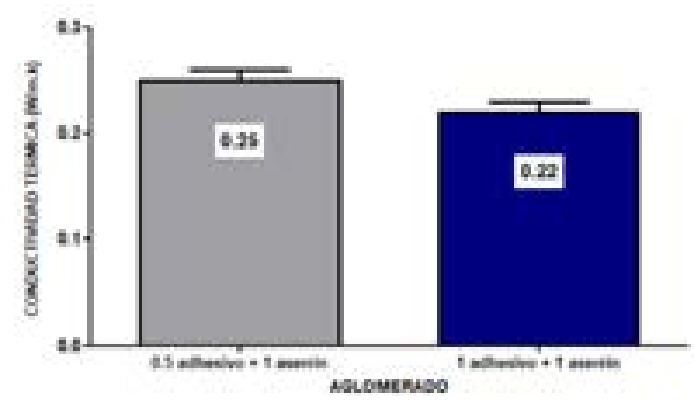

Fig. 2: Conductividad térmica según mezcla del aglomerado. Se muestra que la mezcla con 0.5 de adhesivo de viruta wet blue y 1 parte de aserrín de tornillo presentó 0.25 $\mathrm{W} / \mathrm{m}$.k, mientras que la segunda mezcla con 1 parte de adhesivo de viruta de wet blue y 1 parte de aserrín con $0.22 \mathrm{~W} / \mathrm{m} . \mathrm{k}$.

\section{Densidad aparente del aglomerado}

La densidad aparente para mezclas de los aglomerados presentaron diferencias altamente significativas $(p<0.01)$, estando los promedios de los aglomerados entre los valores de $0.560 \mathrm{gr} / \mathrm{cm} 3$ y $0.900 \mathrm{gr} / \mathrm{cm} 3$ correspondiente a la categoría de aglomerado de alta densidad. Estos tableros son utilizados para construcción en exteriores (tabla 3).

Tabla 3: Comparación de la densidad aparente según mezcla del aglomerado.

\begin{tabular}{|c|c|c|c|c|}
\hline \multirow{3}{*}{ VARIABLE } & \multicolumn{2}{|c|}{ RELACIÓN } & \multirow{3}{*}{$\mathrm{t}$} & \multirow{3}{*}{$P$} \\
\hline & $\begin{array}{c}(0.5 \text { adhesivo }+1 \\
\text { aserrín })\end{array}$ & $\begin{array}{c}(1 \text { adhesivo }+1 \\
\text { aserrín })\end{array}$ & & \\
\hline & $\bar{x} \pm S$ & $\bar{x} \pm s$ & & \\
\hline $\begin{array}{c}\text { Densidad } \\
\text { aparente }(\mathrm{gr} / \\
\mathrm{cm} 3)\end{array}$ & $0.81 \pm 0,02$ & $0.85 \pm 0,02$ & 3.74 & $\begin{array}{c}0.02 \\
(\mathrm{p}<0.05)\end{array}$ \\
\hline
\end{tabular}

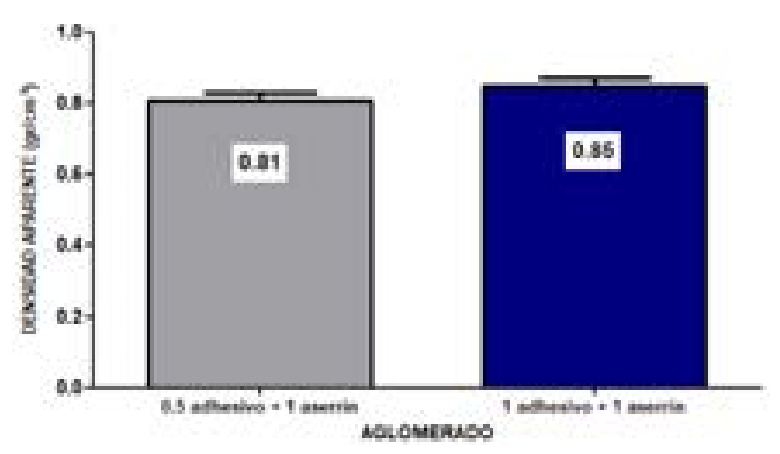

Fig. 3: Densidad aparente según mezcla del aglomerado. Se muestra que la mezcla con 0.5 de adhesivo de viruta wet blue y 1 parte de aserrín de tornillo presentó $0.81 \mathrm{gr} / \mathrm{cm} 3$, mientras que la segunda mezcla con 1 parte de adhesivo de viruta de wet blue y 1 parte mientras que la segunda me
de aserrín con $0.85 \mathrm{gr} / \mathrm{cm} 3$.

\section{CONCLUSIÓN}

La conductividad térmica que la mezcla 1/1 (250gr de adhesivo y 250 gr de aserrín), presentó menor promedio que la norma ASTM C-177 $(0.28 \mathrm{~W} / \mathrm{m} . \mathrm{K})$ con un promedio de $0.22 \mathrm{~W} / \mathrm{m} . \mathrm{K}$, además esta mezcla presentó mayor resistencia a la compresión que la norma DIN $68763(175 \mathrm{Kgf} / \mathrm{m} 2)$ con un promedio de $291.57 \mathrm{Kgf} / \mathrm{m} 2$, demostrando ser un aglomerado muy resistente para construcción y representando una solución ecoamigable para la disposición final de la viruta wet blue, del proceso del curtido de pieles.

\section{REFERENCIAS BIBLIOGRÁFICAS}

1. ASTM (American Society for Testing and Materials). 1993. Standards Test Methods for Thermal Conductivity of Solids by Means of the Guarded - Comparative Longitudinal Heat Flow Technique. Philadelphia, P A.

2. Alfredo Schneider, Hugo Flores Juan Carlos Retamar, Silvio Orué, Eliana Belis, Albano Lacoste. Aglomerado de virutas de cuero. influencia de la presión de moldeo sobre sus propiedades. VII Congreso de Medio Ambiente /AUGM. UNLP, La Plata Argentina 2012.

3. Landrock AH. 1985. Adhesives technology handbook. Ed. Noyes, New Jersey.

4. Schneider A, Flores H, Rodi E, Elizalde E \& Sian L. 2009. Valorización de Virutas de Cuero. Comité de publicaciones Congreso Latinoamericano Ciencias Ambientales COPIME 2009. 